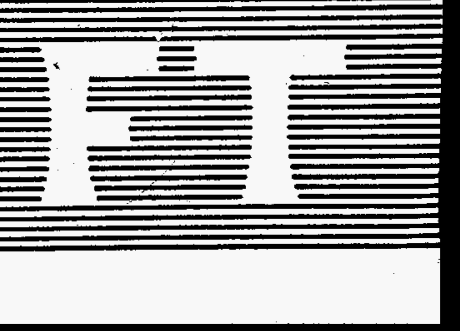

$$
\begin{aligned}
& \text { CONF-9606303--1 } \\
& \text { CONF- } 9609311--1
\end{aligned}
$$

Institute for Fundamental Theory Preprint UFIFT

DOE/ER/40272--260

hep-ph/9611339

\title{
DARK MATTER AXIONS '96
}

\author{
Pierre Sikivie \\ Department of Physics \\ University of Florida \\ Gainesville, FL 32611
}

\section{Contents:}

1. The strong CP problem

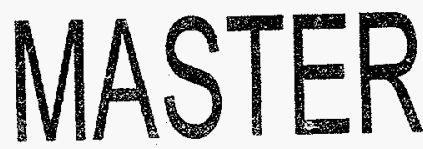

2. Dark matter axions

3. The cavity detector of galactic halo axions

4. The phase space structure of cold dark matter halos

DISTABITAM OF TUIS DOMHENT IS UNLIMIED

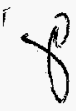

\footnotetext{
*Invited talk at the APCTP Inauguration Conference in Seoul, Korea, June 4-10, 1996 and at the Workshop on Aspects of Dark Matter in Astro- and Particle Physics in Heidelberg, Germany, Sept. 16-20, 1996.
} 


\section{DISCLAIMER}

Portions of this document may be illegible in electronic image products. Images are produced from the best available original document. 


\section{The strong CP problem}

The axion was postulated nearly two decades ago [1] to explain why the strong interactions conserve $P$ and $C P$ in spite of the fact that the weak interactions violate those symmetries. Consider the Lagrangian of QCD:

$$
\mathcal{L}_{Q C D}=-\frac{1}{4} G_{\mu \nu}^{a} G^{a \mu \nu}+\sum_{j=1}^{n}\left[\bar{q}_{j} \gamma^{\mu} i D_{\mu} q_{j}-\left(m_{j} q_{L j}^{+} q_{R j}+\text { h.c. }\right)\right]+\frac{\theta g^{2}}{32 \pi^{2}} G_{\mu \nu}^{a} \tilde{G}^{a \mu \nu}
$$

The last term is a 4-divergence and hence does not contribute in perturbation theory. That term does however contribute through non-perturbative effects [2] associated with QCD instantons [3]. Such effects can make the physics of QCD depend upon the value of $\theta$. Using the Adler-Bell-Jackiw anomaly [4], one can show that $\theta$ dependence must be there if none of the current quark masses vanishes. (If this $\theta$ dependence were absent, QCD would have a $U_{A}(1)$ symmetry and would predict the mass of the $\eta^{\prime}$ pseudo-scalar meson to be less than $\sqrt{3} m_{\pi} \approx 240 \mathrm{MeV}$ [5], contrary to observation.) One can further show that QCD depends upon $\theta$ only through the combination of parameters:

$$
\bar{\theta}=\theta-\arg \left(m_{1}, m_{2}, \ldots m_{n}\right)
$$

If $\bar{\theta} \neq 0, \mathrm{QCD}$ violates $P$ and $C P$. The absence of $P$ and $C P$ violations in the strong interactions therefore places an upper limit upon $\bar{\theta}$. The best constraint follows from the experimental bound [6] on the neutron electric dipole moment which yields: $\bar{\theta}<10^{-9}$.

The question then arises: why is $\bar{\theta}$ so small? In the standard model of particle interactions, the quark masses originate in the electroweak sector of the theory. This sector must violate $P$ and $C P$ to produce the correct weak interaction phenomenology. There is no reason in the standard model to expect the overall phase of the quark mass matrix to exactly match the value of $\theta$ from the QCD sector so that $\bar{\theta}<10^{-9}$. In particular, if $C P$ violation is introduced in the manner of Kobayashi and Maskawa [7], the Yukawa couplings that give masses to the quarks are arbitrary complex numbers and hence arg det $m_{q}$ and $\bar{\theta}$ have no reason to take on any special value at all.

The problem why $\bar{\theta}<10^{-9}$ is usually referred to as the "strong $C P$ problem". The existence of an axion would solve this problem. But, before we talk about axion physics, let's mention that there are other solutions. Setting $m_{u}=0$ removes the $\theta$-dependence of QCD and hence the strong $C P$ problem as well. However, $m_{u}=0$ may cause problems with the successful current algebra relations among pseudo-scalar meson masses. I refer the reader to refs.[8, 9] for recent discussions of the issues involved. Another type of solution involves the assumption that $C P$ and/or $P$ is spontaneously broken but is otherwise a good symmetry. In this case, $\bar{\theta}$ is calculable and may be arranged to be small [10]. Finally, let's emphasize that the strong $C P$ problem need not be solved in the low energy theory. Indeed, as Ellis and Gaillard [11] pointed out, if in the standard model $\bar{\theta}=0$ near the Planck scale then $\bar{\theta} \ll 10^{-9}$ at the QCD scale.

Peccei and Quinn [12] proposed to solve the strong $C P$ problem by postulating the existence of a global $U_{P Q}(1)$ quasi-symmetry. To do its job, $U_{P Q}(1)$ must be a symmetry of the theory at the classical (i.e., at the Lagrangian) level, it must be broken explicitly by 
those non-perturbative effects that make the physics of QCD depend upon $\theta$, and finally it must be spontaneously broken. The axion [13] is the pseudo-Nambu-Goldstone boson associated with the spontaneous breakdown of $U_{P Q}(1)$. One can show that, if a $U_{P Q}(1)$ quasi-symmetry is present, then

$$
\bar{\theta}=\theta-\arg \left(m_{1} \ldots m_{n}\right)-\frac{a(x)}{f_{a}},
$$

where $a(x)$ is the axion field and $f_{a}$, called the axion decay constant, is of order the vacuum expectation value (VEV) which spontaneously breaks $U_{P Q}(1)$. It can further be shown [14] that the non-perturbative effects that make QCD depend upon $\bar{\theta}$ produce an effective potential $V(\bar{\theta})$ whose minimum is at $\bar{\theta}=0$. Thus, by postulating an axion, $\bar{\theta}$ is allowed to relax to zero dynamically and the strong $C P$ problem is solved.

The properties of the axion can be derived using the methods of current algebra [15]. The axion mass is given in terms of $f_{a}$ by

$$
m_{a} \simeq 0.6 \mathrm{eV} \frac{10^{7} \mathrm{GeV}}{f_{a}} .
$$

All the axion couplings are inversely proportional to $f_{a}$. For example, the axion coupling to two photons is:

$$
\mathcal{L}_{a r r}=-g_{r} \frac{\alpha}{\pi} \frac{a(x)}{f_{a}} \vec{E} \cdot \vec{B}
$$

where $\vec{E}$ and $\vec{B}$ are the electric and magnetic fields, $\alpha$ is the fine structure constant, and $g_{\gamma}$ is a model-dependent coefficient of order one. $g_{\gamma}=0.36$ in the DFSZ model [16] whereas $g_{\gamma}=-0.97$ in the KSVZ model [17]. A priori the value of $f_{a}$, and hence that of $m_{a}$, is arbitrary. However, searches for the axion in high energy and nuclear physics experiments combined with astrophysical constraints, the latter derived by considering the effect of the axion upon the lifetimes of red giants and SN1987a, rule out $m_{a} \gtrsim 10^{-3} \mathrm{eV}[1]$. In addition, as will be discussed in section II, cosmology places a lower limit on $m_{a}$ of order $10^{-6} \mathrm{eV}$ by requiring that axions do not overclose the universe.

\section{Dark matter axions}

For small masses, axion production in the early universe is dominated by a novel mechanism [18]. The crucial point is that the non-perturbative QCD effects that produce the effective potential $V(\bar{\theta})$ are strongly suppressed at temperatures high compared to $\Lambda_{Q C D}[19]$. At these high temperatures, the axion is massless and all values of $\langle a(x)\rangle$ are equally likely. At $T \simeq 1 \mathrm{GeV}$, the potential $V$ turns on and the axion field starts to oscillate about a $C P$ conserving minimum of $V$. These oscillations do not dissipate into other forms of energy because, in the relevant mass range, the axion is too weakly coupled for that to happen. The oscillations of the axion field may be described as a fluid of axions. The typical momentum of the axions in the fluid is the inverse of the correlation length of the axion field. Because that correlation length is of order the horizon, we have $p_{a} \sim\left(10^{-6}\right.$ 
sec $)^{-1} \sim 10^{-9} \mathrm{eV}$ at $T \simeq 1 \mathrm{GeV}$, and $p_{a} \sim R^{-1}$ afterwards. $R$ is the cosmological scale factor here. Thus the axion fluid is very cold compared to the ambient temperature.

Let me briefly indicate how the present cosmological energy density of this axion fluid is estimated. Let $\varphi(x)$ be the complex scalar field whose VEV $v$ spontaneously breaks $U_{P Q}(1)$. At extremely high temperatures, the $U_{P Q}(1)$ symmetry is restored. It becomes spontaneously broken when the temperature drops below a critical value $T_{P Q}$ of order $v$. Below $T_{P Q}$, the axion field $a(x)$ appears as the phase of the $V E V$ of $\varphi:\langle\varphi(x)\rangle=v e^{i a(x) / v}$. We must now distinguish two cases. Either inflation occurs with reheat temperature below $T_{P Q}$, or not (i.e., inflation does not occur or it occurs with reheat temperature above $T_{P Q}$ ). In the first case, inflation homogenizes the axion field and there is only one contribution to the axion cosmological energy density, the contribution from so-called "vacuum misalignment". In the second case, there are additional contributions from axion string and axion domain wall decay. Only the contribution from vacuum misalignment is discussed in any detail here.

When the axion mass turns on near the QCD phase transition, the axion field starts to oscillate about one of the $C P$ conserving minima of the effective potential. The oscillation begins approximately at cosmological time $t_{1}$ such that $t_{1} m_{a}\left(T\left(t_{1}\right)\right)=0(1)$, where $m_{a}(T)$ is the temperature dependent axion mass. Soon after $t_{1}$, the axion mass changes sufficiently slowly that the total number of axions in the oscillations of the axion field is an adiabatic invariant. $T_{1} \equiv T\left(t_{1}\right)$ has been estimated to be of order $1 \mathrm{GeV}$. The number density of axions at time $t_{1}$ is

$$
n_{a}\left(t_{1}\right) \simeq \frac{1}{2} m_{a}\left(t_{1}\right)\left\langle a^{2}\left(t_{1}\right)\right\rangle \simeq \pi f_{a}^{2} \frac{1}{t_{1}}
$$

where $f_{a}=\frac{v}{N}$ is the axion decay constant introduced earlier. $N$ is an integer which expresses the color anomaly of $U_{P Q}(1) . \quad N$ also equals the number of $C P$ conserving vacua [20] at the bottom of the 'Mexican hat' potential, i.e., in the interval $0 \leq \frac{a}{v}<2 \pi$. In Eq. (2.1), we used the fact that the axion field $a(x)$ is approximately homogeneous on the horizon scale $t_{1}$. Wiggles in $a(x)$ which entered the horizon long before $t_{1}$ have been red-shifted away [21]. We also used the fact that the initial departure of $a(x)$ from the nearest minimum is of order $\frac{v}{N}=f_{a}$. The axions of Eq. (2.1) are decoupled and nonrelativistic. Assuming that the ratio of the axion number density to the entropy density is constant from time $t_{1}$ till today, one finds [18]

$$
\Omega_{a}=\left(\frac{0.610^{-5} \mathrm{eV}}{m_{a}}\right)^{\frac{7}{6}}\left(\frac{200 \mathrm{MeV}}{\Lambda_{Q C D}}\right)^{\frac{3}{4}}\left(\frac{75 \mathrm{~km} / \mathrm{s} \cdot \mathrm{Mpc}}{H_{0}}\right)^{2}
$$

for the ratio of the axion energy density to the critical density for closing the universe. $H_{0}$ is the present Hubble rate. Eq. (2.2) implies the bound $m_{a} \gtrsim 10^{-6} \mathrm{eV}$.

However, it should be emphasized that there are many sources of uncertainty in the estimate of Eq. (2.2). The axion energy density may be diluted by the entropy release from heavy particles which decouple before the QCD epoch but decay afterwards [22], or by the entropy release associated with a first order QCD phase transition. On the other hand, if the QCD phase transition is first order [23], an abrupt change of the axion mass at the transition may increase $\Omega_{a}$. If inflation occurs with reheat temperature less than $T_{P Q}$, there may be an accidental suppression of $\Omega_{a}$ because the homogenized axion 
field happens to lie close to a $C P$ conserving minimum. Because the RHS of Eq. (2.2) is multiplied in this case by a factor of order the square of the initial vacuum misalignment angle $\frac{a\left(t_{1}\right)}{v} N$ which is randomly chosen between $-\pi$ and $+\pi$, the probability that $\Omega_{a}$ is suppressed by a factor $x$ is of order $\sqrt{x}$. This rule cannot be extended to arbitrarily small $x$ however because quantum mechanical fluctuations in the axion field during the epoch of inflation do not allow the suppression to be perfect [24]. If inflation occurs with reheating temperature larger than $T_{P Q}$ or if there is no inflation, there are contributions to $\Omega_{a}$ from axion string [25] and axion domain wall decay in addition to the contribution, Eq. (2.2), from vacuum misalignment. The author and his collaborators [26] have estimated each of these additional contributions to be of the same order of magnitude as that from vacuum misalignment. Others $[25,27]$ have estimated that the contribution from axion string decay dominates over that from vacuum misalignment by a factor 100 or a factor 10 .

The axions produced when the axion mass turns on during the QCD phase transition are cold dark matter (CDM) because the axions are non-relativistic from the moment of their first appearance at $1 \mathrm{GeV}$ temperature. Studies of large scale structure formation support the view that the dominant fraction of dark matter is CDM [28]. Moreover. any form of CDM necessarily contributes to galactic halos by falling into the gravitational wells of galaxies. Hence, there is excellent motivation to look for CDM candidates as constituent particles of our galactic halo, even after some fraction of our halo has been demonstrated to be in MACHOs [29] or some other form.

Finally, let's mention that there is a particular kind of clumpiness [30] which affects axion dark matter if there is no inflation after the Peccei-Quinn phase transition. This is due to the fact that the dark matter axions are inhomogeneous with $\delta \rho / \rho \sim 1$ over the horizon scale at temperature $T_{1} \simeq 1 \mathrm{GeV}$, when they are produced at the start of the QCD phase-transition, combined with the fact that their velocities are so small that they do not erase these inhomogeneities by free-streaming before the time $t_{e q}$ of equality between the matter and radiation energy densities when matter perturbations can start to grow. These particular inhomogeneities in the axion dark matter are immediately in the non-linear regime after time $t_{e q}$ and thus form clumps, called 'axion mini-clusters' [30]. These have mass $M_{m c} \simeq 10^{-13} M_{\odot}$ and size $l_{m c} \simeq 10^{12} \mathrm{~cm}$.

\section{The cavity detector of galactic halo axions}

Axions can be detected by stimulating their conversion to photons in a strong magnetic field [31]. The relevant coupling is given in Eq. (1.5). In particular, an electromagnetic cavity permeated by a strong static magnetic field can be used to detect galactic halo axions. The latter have velocities $\beta$ of order $10^{-3}$ and hence their energies $E_{a}=m_{a}+\frac{1}{2} m_{a} \beta^{2}$ have a spread of order $10^{-6}$ above the axion mass. When the frequency $\omega=2 \pi f$ of a cavity mode equals $m_{a}$, galactic halo axions convert resonantly into quanta of excitation (photons) of that cavity mode. The power from axion $\rightarrow$ photon conversion on resonance is found to be $[31,32]$ :

$$
P=\left(\frac{\alpha}{\pi} \frac{g_{\gamma}}{f_{a}}\right)^{2} V B_{0}^{2} \rho_{a} C \frac{1}{m_{a}} \operatorname{Min}\left(Q_{L}, Q_{a}\right)
$$




$$
\begin{aligned}
& =0.510^{-26} \text { Watt }\left(\frac{V}{500 \text { liter }}\right)\left(\frac{B_{0}}{7 \text { Tesla }}\right)^{2} C\left(\frac{g_{\gamma}}{0.36}\right)^{2} \\
& \cdot\left(\frac{\rho_{a}}{\frac{1}{2} \cdot 10^{-24} \frac{g_{r}}{\mathrm{~cm}^{3}}}\right)\left(\frac{m_{a}}{2 \pi(\mathrm{GHz})}\right) \operatorname{Min}\left(Q_{L}, Q_{a}\right)
\end{aligned}
$$

where $V$ is the volume of the cavity, $B_{0}$ is the magnetic field strength, $Q_{L}$ is its loaded quality factor, $Q_{a}=10^{6}$ is the 'quality factor' of the galactic halo axion signal (i.e. the ratio of their energy to their energy spread), $\rho_{a}$ is the density of galactic halo axions on Earth, and $C$ is a mode dependent form factor given by

$$
C=\frac{\left|\int_{V} d^{3} x \vec{E}_{\omega} \cdot \vec{B}_{0}\right|^{2}}{B_{0}^{2} V \int_{V} d^{3} x \epsilon\left|\vec{E}_{\omega}\right|^{2}}
$$

where $\vec{B}_{0}(\vec{x})$ is the static magnetic field, $\vec{E}_{\omega}(\vec{x}) e^{i \omega t}$ is the oscillating electric field and $\epsilon$ is the dielectric constant.

Because the axion mass is only known in order of magnitude at best, the cavity must be tunable and a large range of frequencies must be explored seeking a signal. The cavity can be tuned by moving a dielectric rod or metal post inside it. Using Eq. (3.1), one finds that to perform a search with signal to noise ratio $s / n$, the scanning rate is:

$$
\begin{aligned}
\frac{d f}{d t}= & \frac{12 \mathrm{GHz}}{\text { year }}\left(\frac{4 n}{s}\right)^{2}\left(\frac{V}{500 \text { liter }}\right)^{2}\left(\frac{B_{0}}{7 \text { Tesla }}\right)^{4} C^{2}\left(\frac{g_{\gamma}}{0.36}\right)^{4} \\
& \cdot\left(\frac{\rho_{a}}{\frac{1}{2} \cdot 10^{-24} \frac{g^{2}}{\mathrm{~cm}^{3}}}\right)^{2}\left(\frac{3 K}{T_{n}}\right)^{2}\left(\frac{f}{\mathrm{GHz}}\right)^{2} \frac{Q_{L}}{Q_{a}}
\end{aligned}
$$

where $T_{n}$ is the sum of the physical temperature of the cavity plus the noise temperature of the microwave receiver that detects the photons from $a \rightarrow \gamma$ conversion. Eq. (3.3) assumes that $Q_{L}<Q_{a}$ and that some strategies have been followed which optimize the search rate. The best quality factors attainable at present, using oxygen free copper, are of order $10^{5}$ in the $\mathrm{GHz}$ range. To make the cavity of superconducting material is probably not useful since it is permeated by a strong magnetic field in the experiment.

Eq. (3.3) shows that a galactic halo search at the required sensitivity is feasible with presently available technology, provided the form factor $C$ can be kept at values of order one for a wide range of frequencies. For a cylindrical cavity and a longitudinal magnetic field, $C=0.69$ for the lowest TM mode. The form factors of the other modes are much smaller. The resonant frequency of the lowest TM mode of a cylindrical cavity is $f=115 \mathrm{MHz}\left(\frac{1 m}{R}\right)$ where $R$ is the radius of the cavity. Since $10^{-6} \mathrm{eV}=2 \pi(242 \mathrm{MHz})$, a large cylindrical cavity is convenient for searching the low frequency end of the range of interest. To extend the search to high frequencies without sacrifice in volume, one may power-combine many identical cavities which fill up the available volume inside a magnet's bore [33, 34]. This method allows one to maintain $C=0(1)$ at high frequencies, albeit at the cost of increasing engineering complexity as the frequency, and hence the number of cavities, is increased.

Pilot experiments were carried out at Brookhaven National Laboratory [35] and at the University of Florida [36]. The (magnetic field) ${ }^{2} \times$ volume provided by the magnets used 


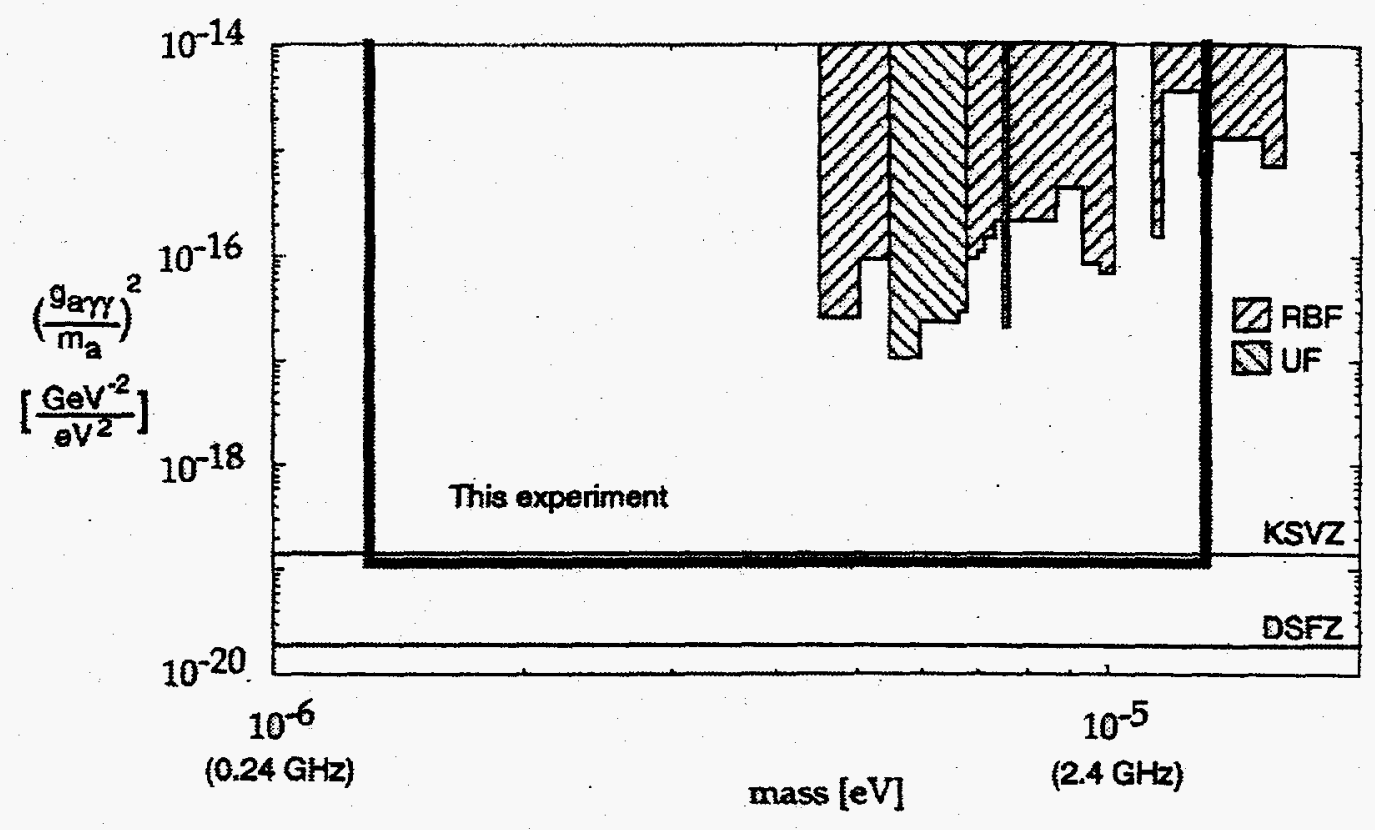

Figure 1: Regions in mass - (coupling/mass) ${ }^{2}$ space which have been ruled out by the RBF and UF experiments (hatched) and which the LLNL experiment expects being able to rule out (shaded border). The relation between coupling and mass in the DFSZ and KSVZ models is also shown. 
in these experiments were relatively low: $B_{0}^{2} V=0.36 T^{2} m^{3}$ and $0.45 T^{2} m^{3}$ respectively for Brookhaven (RBF) and Florida (UF). Fig. I shows the limits that these experiments placed on the square of the coupling $g_{a \gamma \gamma}=\frac{\alpha}{\pi} \frac{g_{\gamma}}{f_{a}}$ as a function of the axion mass $m_{a}$ assuming that the local density of galactic halo axions is $\rho_{a}=\frac{1}{2} \cdot 10^{-24} \mathrm{gr} / \mathrm{cm}^{3}$. The figure also shows $g_{a \gamma \gamma}$ as a function of $m_{a}$ in the KSVZ and DFSZ models.

Second generation experiments are presently under way at Lawrence Livermore National Laboratory (LLNL) [37] and at Kyoto University [38]. The LLNL experiment uses a much larger magnet $\left(B_{0}^{2} V=12 T^{2} m^{3}\right)$ than the pilot experiments. It also improves the noise temperature $\left(T_{n}=3 \mathrm{~K}\right.$ vs. $T_{n}=5 \mathrm{~K}$ for the Florida experiment) although it uses the same microwave receiver technology (HEMT amplifiers). The LLNL experiment is also the first to use multiple cavity arrays to expand widely the mass range searched. It will cover $1.3<m_{a}<13 \mu \mathrm{eV}$ at a level of sensitivity sufficient to discover KSVZ axions if they are the constituents of our galactic halo; see Fig. I. The LLNL experiment started taking data in Feb. '96 and will run for about three years to cover the stated range.

The Kyoto experiment has a magnet of size similar to that of the pilot experiments but uses a beam of Rydberg atoms to count the photons from $a \rightarrow \gamma$ conversion. The $a \rightarrow \gamma$ conversion part is the same as in the other experiments. Single photon counting constitutes a dramatic improvement in microwave detection sensitivity. With HEMT amplifiers one needs to have thousands of $a \rightarrow \gamma$ conversions per second and integrate for about $100 \mathrm{sec}$ to find a signal in the noise. With single photon counting, a few $a \rightarrow \gamma$ conversions suffice in principle. To build a beam of Rydberg atoms capable of single photon counting is a considerable achievement. In addition, the cavity will be cooled by a dilution refrigerator down to a temperature $(\sim 10 \mathrm{mK})$ where the thermal photon background is negligible. The Kyoto experiment will first search near $m_{a}=10^{-5} \mathrm{eV}$. Its projected sensitivity is sufficient to discover DFSZ axions even if their local density is only $\frac{1}{5}$ of the local halo density.

\section{The phase space structure of cold dark matter halos}

If a signal is found in the cavity detector of galactic halo axions, it will be possible to measure their energy spectrum with great precision and resolution because all the time previously used in searching for the signal can now be used to accumulate data. Hence there is good motivation to ask what can be learned about our galaxy from analyzing such a signal.

In many past discussions of dark matter detection on Earth, it has been assumed that the dark matter particles have an isothermal distribution. Thermalization has been argued to be the result of a period of "violent relaxation" following the collapse of the protogalaxy. If it is strictly true that the velocity distribution of dark matter particles is isothermal, which seems to be a strong assumption, then the only information that can be gained from its observation is the corresponding virial velocity and our own velocity relative to its standard of rest. If, on the other hand, thermalization is incomplete, a signal in a dark matter detector may yield additional information.

J.R. lpser and I discussed [39] the extent to which the phase-space distribution of 


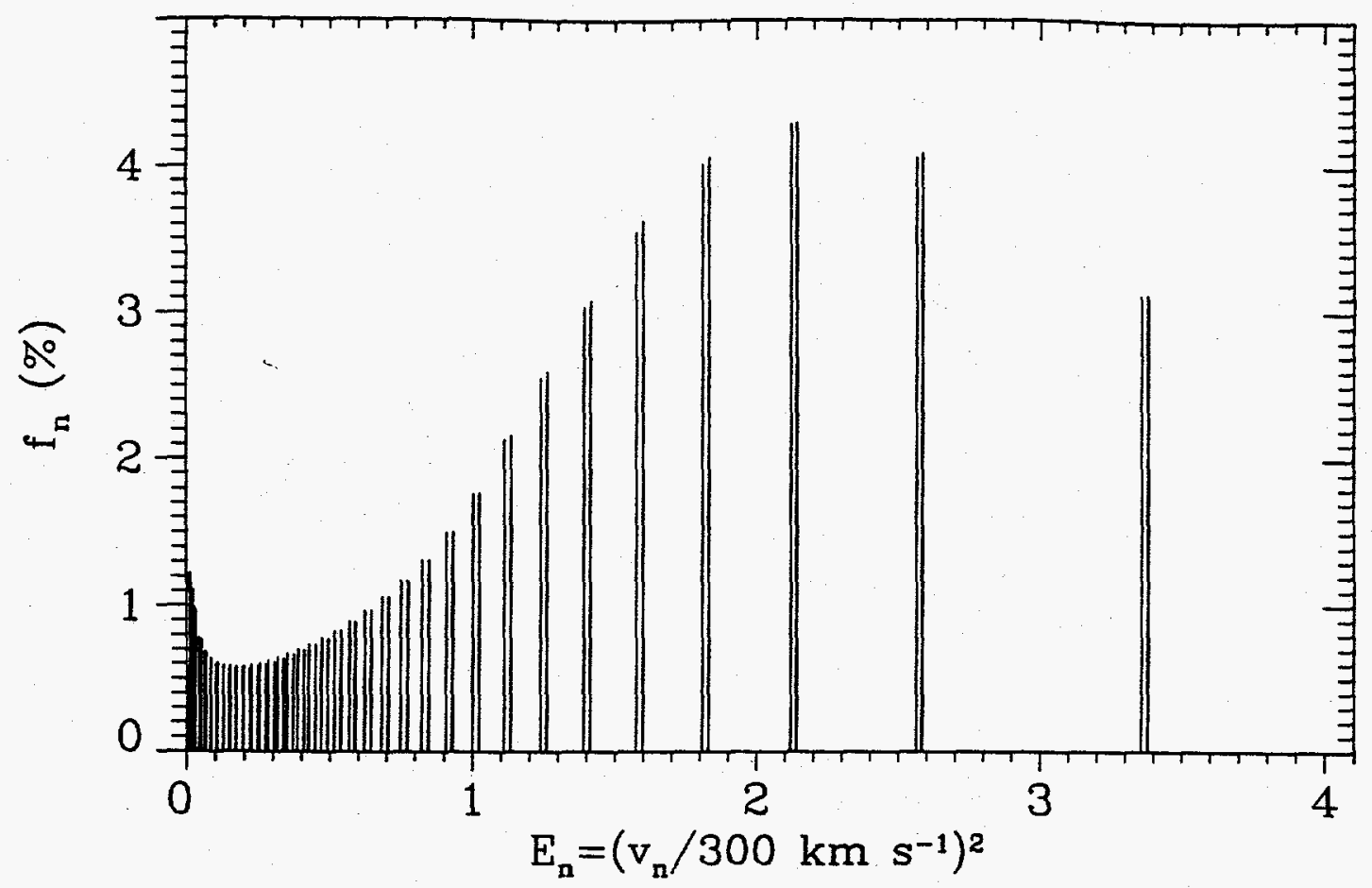

Figure 2: The spectrum of velocity peaks in a typical case $(\epsilon=0.2, h=0.7$ and $\bar{j}=0.2)$ studied in ref. [40]. $f_{n}$ and $E_{n}$ are defined in the text.

cold dark matter particles is thermalized in a galactic halo and concluded that there are substantial deviations from a thermal distribution in that the highest energy particles have discrete values of velocity. There is one velocity peak on Earth due to dark matter particles falling onto the galaxy for the first time, one peak due to particles falling out of the galaxy for the first time, one peak due to particles falling into the galaxy for the second time, etc. The peaks due to particles that have fallen in and out of the galaxy a large number of times in the past are washed out because of scattering in the gravitational wells of stars, globular clusters and large molecular clouds. But the peaks due to particles which have fallen in and out of the galaxy only a small number of times in the past are not washed out.

I. Tkachev, Y. Wang and I have used the secondary infall model of galactic halo formation to estimate the local densities and the velocity magnitudes of the dark matter particles in the velocity peaks [40]. We generalized the existing version of that model to take account of the angular momentum of the dark matter particles. In the absence of angular momentum, the model produces flat rotation curves for a large range of values of a parameter $\epsilon$ whose average value may be inferred from the spectrum of primordial density perturbations. We find that the presence of angular momentum produces an effective core radius, i.e., it makes the contribution of the halo to the rotation curve go to zero at zero radius. The model provides a detailed description of the large scale properties of galactic halos including their density profiles, their extent and their total mass. Fig. II shows the predictions of the model for the average density fractions $f_{n}=\rho_{n} / \rho$ and the 
kinetic energies per unit mass $E_{n}$ of the particles in the highest energy peaks for the case where $\epsilon=0.2, H_{0}=70 \mathrm{~km} / \mathrm{sec} \cdot \mathrm{Mpc}$ and the average amount of angular momentum, in the dimensionless units defined in ref. [40], is $\bar{j}=0.2$. The density fractions $f_{n}$ are averages over all locations at the same distance $(8.5 \mathrm{kpc})$ from the galactic center as we are. The $E_{n}$ are measured in a frame of reference which is not co-rotating with the disk.

\section{Acknowledgements:}

I thank the Aspen Center for Physics for its hospitality while writing up this lecture. This work is supported in part by the US Department of Energy under grant No. DEFG0586ER40272.

\section{References}

[1] Axion reviews include: J.E. Kim, Phys. Rep. 150 (1987) 1; H.-Y. Cheng, Phys. Rep. 158 (1988) 1; R.D. Peccei, in 'CP Violation', ed. by C. Jarlskog, World Scientific Publ., 1989, pp 503-551; M.S. Turner, Phys. Rep. 197 (1990) 67; G.G. Raffelt, Phys. Rep. 198 (1990) 1.

[2] G. 't Hooft, Phys: Rev. Lett. 37 (1976) 8 and Phys. Rev. D14 (1976) 3432; R. Jackiw and C. Rebbi, Phys. Rev. Lett. 37 (1976) 172; C. G. Callan, R. F. Dashen and D. J. Gross, Phys. Lett. B63 (1976) 334.

[3] A.A Belavin, A.M. Polyakov, A.S. Shvarts and Yu.S. Tyupkin, Phys. Lett. 59B (1975) 85.

[4] S. Adler, Phys. Rev. 177 (1969) 2426; J.S. Bell and R. Jackiw, Nuov. Cim. 60A (1969) 47.

[5] S. Weinberg, Phys. Rev. D11 (1975) 3583.

[6] I.S. Altarev et al., Phys. Lett. B276 (1992) 242; K.F. Smith et al., Phys. Lett. B234 (1990) 191.

[7] M. Kobayashi and K. Maskawa, Progr. Theor. Phys. 49 (1973) 652.

[8] T. Banks, Y. Nir and N. Seiberg, hep-ph/9403203, in the Proceedings of the 2nd IFT Workshop,'Yukawa couplings and the origin of mass', Gainesville, Florida, Feb. 1994, ed. P. Ramond, International Press, Cambridge, MA, 1995.

[9] H. Leutwyler, hep-ph/9602255, to be published in the Proceedings of the Conference on Fundamental Interactions of Elementary Particles, ITEP, Moscow, Russia, Oct. 1995. 
[10] M.A.B. Beg and H.S. Tsao, Phys. Rev. Lett. 41 (1978) 278; R.N. Mohapatra and G. Senjanovic, Phys. Lett. B79 (1978) 283; H. Georgi, Hadronic J. 1 (1978) 155; A.E. Nelson, Phys. Lett. B136 (1984) 387; S.M. Barr, Phys. Rev. D30 (1984) 1805; K.S. Babu and R.N. Mohapatra, Phys. Rev. D41 (1990) 1286; S.M. Barr, D. Chang and G. Senjanovic, Phys. Rev. Lett. 67 (1991) 2765; R. Kuchimanchi, Phys. Rev. Lett. 76 (1996) 3486; R.N. Mohapatra and A. Rasin, Phys. Rev. Lett. 76 (1996) 3490.

[11] J. Ellis and M.K. Gaillard, Nucl. Phys. B150 (1979) 141.

[12] R. D. Peccei and H. Quinn, Phys. Rev. Lett. 38 (1977) 1440 and Phys. Rev. D16 (1977) 1791.

[13] S. Weinberg, Phys. Rev. Lett. 40 (1978) 223; F. Wilczek, Phys. Rev. Lett. 40 (1978) 279.

[14] C. Vafa and E. Witten, Phys. Rev. Lett. 53 (1984) 535.

[15] W.A. Bardeen and S.-H.H. Tye, Phys. Lett. B74 (1978) 229; J. Ellis and M.K. Gaillard, Nucl. Phys. B150 (1979) 141; T.W. Donnelly et al., Phys. Rev. D18 (1978) 1607; M. Srednicki, Nucl. Phys. 260 (1985) 689; P. Sikivie, in 'Cosmology and Particle Physics', ed. E. Alvarez et al., World Scientific, 1987, pp 143-169.

[16] M. Dine, W. Fischler and M. Srednicki, Phys. Lett. B104 (1981) 199; A. P. Zhitnitskii, Sov. J. Nucl. 31 (1980) 260.

[17] J. Kim, Phys. Rev. Lett. 43 (1979) 103; M. A. Shifman, A. I. Vainshtein and V. I. Zakharov, Nucl. Phys. B166 (1980) 493.

[18] L. Abbott and P. Sikivie, Phys. Lett. B120 (1983) 133; J. Preskill, M. Wise and F. Wilczek, Phys. Lett. B120 (1983) 127; M. Dine and W. Fischler, Phys. Lett. B120 (1983) 137.

[19] D. J. Gross, R. D. Pisarski and L. G. Yaffe, Rev. Mod. Phys. 53 (1981) 43.

[20] P. Sikivie, Phys. Rev. Lett. 48 (1982) 1156.

[21] A. Vilenkin, Phys. Rev. Lett. 48 (1982) 59.

[22] P. J. Steinhardt and M. S. Turner, Phys. Lett. B129 (1983) 51; G. Lazarides, R. Schaefer, D. Seckel and Q. Shafi, Nucl. Phys. B346 (1990) 193.

[23] W. G. Unruh and R. M. Wald, Phys. Rev. D32 (1985) 831; M. S. Turner, Phys. Rev. D32 (1985) 843; T. DeGrand, T. W. Kephart and T. J. Weiler, Phys. Rev. D33 (1986) 910; M. Hindmarsh, Phys. Rev. D45 (1992) 1130.

[24] A. D. Linde, JETP Lett. 40 (1984) 1333 and Phys. Lett. B158 (1985) 375; D. Seckel and M. Turner, Phys. Rev. D32 (1985) 3178; D. H. Lyth, Phys. Lett. B236 (1990) 408; A. D. Linde and D. H. Lyth, Phys. Lett. B246 (1990) 353; M. Turner and F. Wiczek, Phys. Rev. Lett. 66 (1991) 5; A. Linde, Phys. Lett. B259 (1991) 38; D.H. 
Lyth, Phys. Rev. D45 (1992) 3394; D.H. Lyth and E.D. Stewart, Phys. Lett. B283 (1992) 189 and Phys. Rev. D46 (1992) 532.

[25] R. Davis, Phys. Rev. D32 (1985) 3172 and Phys. Lett. B180 (1986) 225.

[26] D. Harari and P. Sikivie, Phys. Lett. B195 (1987) 361; C. Hagmann and P. Sikivie, Nucl. Phys. B363 (1991) 247.

[27] A. Vilenkin and T. Vachaspati, Phys. Rev. D35 (1987) 1138; R.L. Davis and E.P.S. Shellard, Nucl. Phys. B324 (1989) 167; A. Dabholkar and J. Quashnock, Nucl. Phys. B333 (1990) 815; R.A. Battye and E.P.S. Shellard, Nucl.Phys.B423 (1994) 260, Phys. Rev. Lett. 73 (1994) 2954 and erratum-ibid. 76 (1996) 2203.

[28] S. Dodelson, E. Gates and M.S. Turner, astro-ph/9603081.

[29] C. Alcock et al., Phys. Rev. Lett. 74 (1995) 2867, and astro-ph/9606165.

[30] C. J. Hogan and M. J. Rees, Phys. Lett. B205 (1988) 228; E. Kolb and I. I. Tkachev, Phys. Rev. Lett. 71 (1993) 3051, Phys. Rev. D49 (1994) 5040, and Ap. J. 460 (1996) L25.

[31] P. Sikivie, Phys. Rev. Lett. 51 (1983) 1415 and Phys. Rev. D32 (1985) 2988.

[32] L. Krauss, J. Moody, F. Wilczek and D. Morris, Phys. Rev. Lett. 55 (1985) 1797.

[33] C. Hagmann et al., Rev. Sci. Inst. 61 (1990) 1076.

[34] C. Hagmann, Ph. D. thesis, unpublished.

[35] S. DePanfilis et al., Phys. Rev. Lett. 59 (1987) 839 and Phys. Rev. 40 (1989) 3153.

[36] C. Hagmann et al., Phys. Rev. D42 (1990) 1297.

[37] K. van Bibber et al., Int. J. Mod. Phys. D3 Suppl.(1994) 33.

[38] S. Matsuki and K. Yamamoto, Phys. Lett. B263 (1991) 523; S. Matsuki, I. Ogawa, K. Yamamoto, Phys. Lett. B336 (1994) 573.

[39] J.R. Ipser and P. Sikivie, Phys. Lett. B291 (1992) 288.

[40] P. Sikivie, I.I. Tkachev and Y. Wang, Phys. Rev. Lett. 75 (1995) 2911 and astro$\mathrm{ph} / 9609022$. 